\title{
Light Environment Design to Enhance the Uniformity of Strawberry Canopy Lighting in Greenhouse
}

\author{
Xiaoyang $\mathrm{He}^{1,2,3^{*}}$, Yan Liu ${ }^{3}$, Qipeng $\mathrm{He}^{4}$, Chenyu Zhao ${ }^{3}$, Haoyu $\mathrm{Hu}^{3}$, Jinshuai Wang ${ }^{3}$, Xiao \\ $\mathrm{Li}^{3}$, Ling $\mathrm{Yu}^{3 *}$ \\ \{hexy@dlpu.edu.cn,1258570169@qq.com,hqpdyx@163.com\} \\ University of Chinese Academy of Sciences,Beijing,100049, China ${ }^{1}$, Shenyang Institute of Computing \\ Technology Chinese academy of sciences, Shenyang, Liaoning Province,110168,China ${ }^{2}$,Dalian Polytechnic \\ University, Dalian, Liaoning Province,116034,China ${ }^{3}$,Guangchuang service center of Guizhou Zhifu Optical \\ Valley Investment Management Co., Ltd. ${ }^{4}$
}

\begin{abstract}
Under different installation methods, the illumination uniformity of strawberry canopy was tested, and the light environment was simulated by dialux evo. Through the simulation test of different arrangement, installation height and luminous angle, the data of three different working faces are observed. Through analysis and calculation, a kind of light environment design which can improve the illumination uniformity of strawberry canopy in greenhouse is obtained.
\end{abstract}

Keywords: illumination uniformity; light environment design; plant lighting; plant factory;

\section{Introduction}

With the development of lighting technology, people are no longer satisfied with the growth of plants under the sun, but supplement the light of plants. In some areas with bad climate, The efficient plant lighting technology can shorten plant growth cycle and increase crop quality. A LED lamp containing a spectrum suitable for plant growth acts on the growing plant to make up for the lack of light caused by environmental reasons, which can not only promote its growth, but also improve the quality of the plant. 
Because the leaf distribution of plants is not uniform, under certain light conditions, the top leaf reaches saturation, but the bottom leaf does not reach light saturation at this time, the illumination will be uneven, which will affect the growth of plants. As a herbaceous plant, the plant height range in the growth cycle is generally $10 \mathrm{~cm}-40 \mathrm{~cm}$, and the number of leaves with different heights varies greatly. Under the condition of natural light, it is easy to produce the above uneven illumination, which is suitable for artificial light supplement experiment ${ }^{[1]}$. Therefore,strawberry was selected as the experimental object. LED light source has the advantages of long service life, using DC, low heating and so on. At the same time, the LED light source system can also regulate the light quality, and can realize close irradiation with crops, which is suitable for this simulation experiment. In the process of experiment, the illumination and evenness of strawberry in vertical direction were studied by adjusting the installation position and luminous angle of lamps and lanterns. Dialux evo is used to simulate the light environment, three working faces with different heights are set up, and the illumination uniformity of the three working faces is calculated. By reducing the difference of illumination in the vertical direction of plant leaves, a suitable light environment for strawberry growth is obtained.

\section{Design principles}

In order to solve the problem that the canopy leaves are affected by uneven light, the illumination difference of strawberry plants in vertical direction is reduced by adjusting the lamp arrangement, installation height and luminous angle, so that the crops can receive light evenly in vertical direction. As a whole, the light distribution mode mainly starts from four aspects :1.illumination 2.illumination uniformity 3.installation capacity reduced to minimum 4.distance to high ratio $(\mathrm{s} / \mathrm{h})^{[2]}$. With different installation height, the lower the installation safety, the greater the illumination uniformity of the working face, so the appropriate intermediate value is found by testing.

The luminous angle of the light source is increased from $20^{\circ}$ to $50^{\circ}$ in $2^{\circ}$. Since the vertical height of mature strawberry plants is not more than $30 \mathrm{~cm}$, three height working faces $(1.3 \mathrm{~m} 、 1.5 \mathrm{~m} 、 1.7 \mathrm{~m})$ are set up in the simulation process to compare the data, and the suitable luminous angle is selected, and the optimal light environment design scheme is obtained. 


\section{Location Design of Light Source}

\subsection{Lamp installation}

The height of plant lighting installation should be determined by the species of vegetables cultivated in solar greenhouse and their botanical and biological characteristics. According to environmental factors, the installation height of the light-filling lamp in greenhouse is 1.9 $\mathrm{m}-3.3 \mathrm{~m}^{[1]}$. By setting this height as the simulation height, it can be seen from the parameters that the lower or higher the installation height is, and the uniformity decreases obviously.'

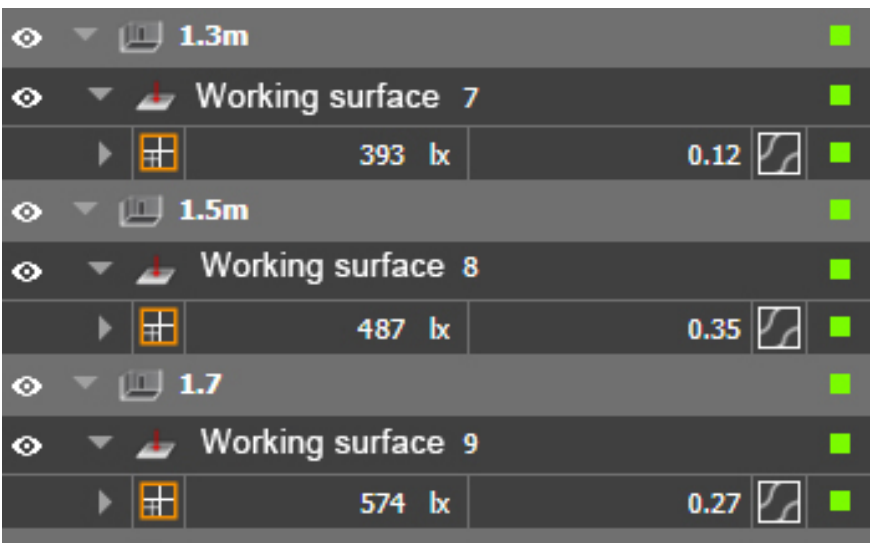

Fig .1. Illumination and illuminance uniformity of the lamp at 2 meters

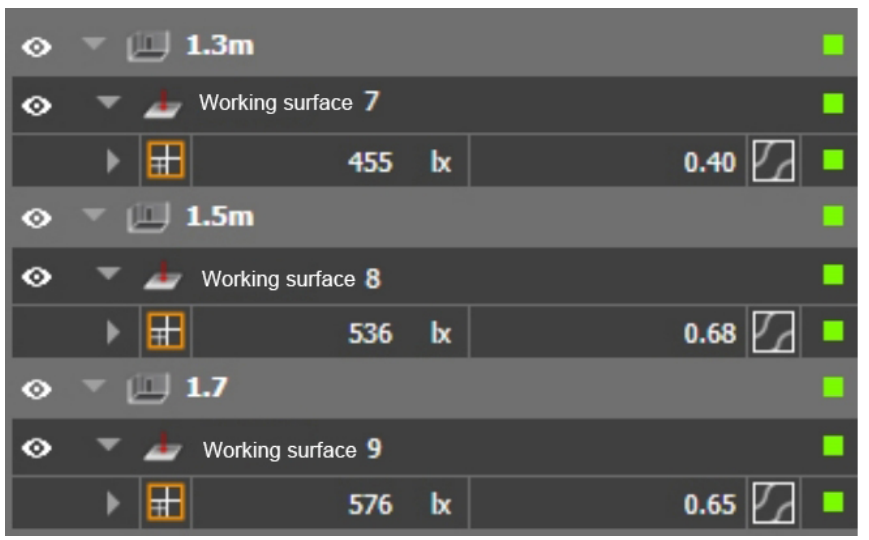


Figure .2. Illumination and illuminance uniformity of the lamp at 2.3 meters

Whether the arrangement of lamps and lanterns is reasonable or not depends on the high ratio $(\mathrm{s} / \mathrm{h})$. When the distance between $\mathrm{s}$ (lamps and lanterns) has been determined $\mathrm{h}$ ( the higher the height of the lamp distance from the working face), the better the illumination uniformity and the poor economy. Therefore, in the early stage of the test, it is found that when the ratio of distance to height is smaller, the difference of parameters between the three working faces is smaller. Therefore, in the similar experimental environment, summarized the existing plant lighting test installation. The lowest installation height and the lowest installation height are obtained, and the simulation test is carried out on the installation of $1.9 \mathrm{~m}-3.3 \mathrm{~m}$ height lamps. The results are as follows: when the ratio of distance to height is 0.48 , the uniformity of the three working faces is the smallest and the highest. The installation height is $2.5 \mathrm{~m}$, so the installation height $2.5 \mathrm{~m}$.

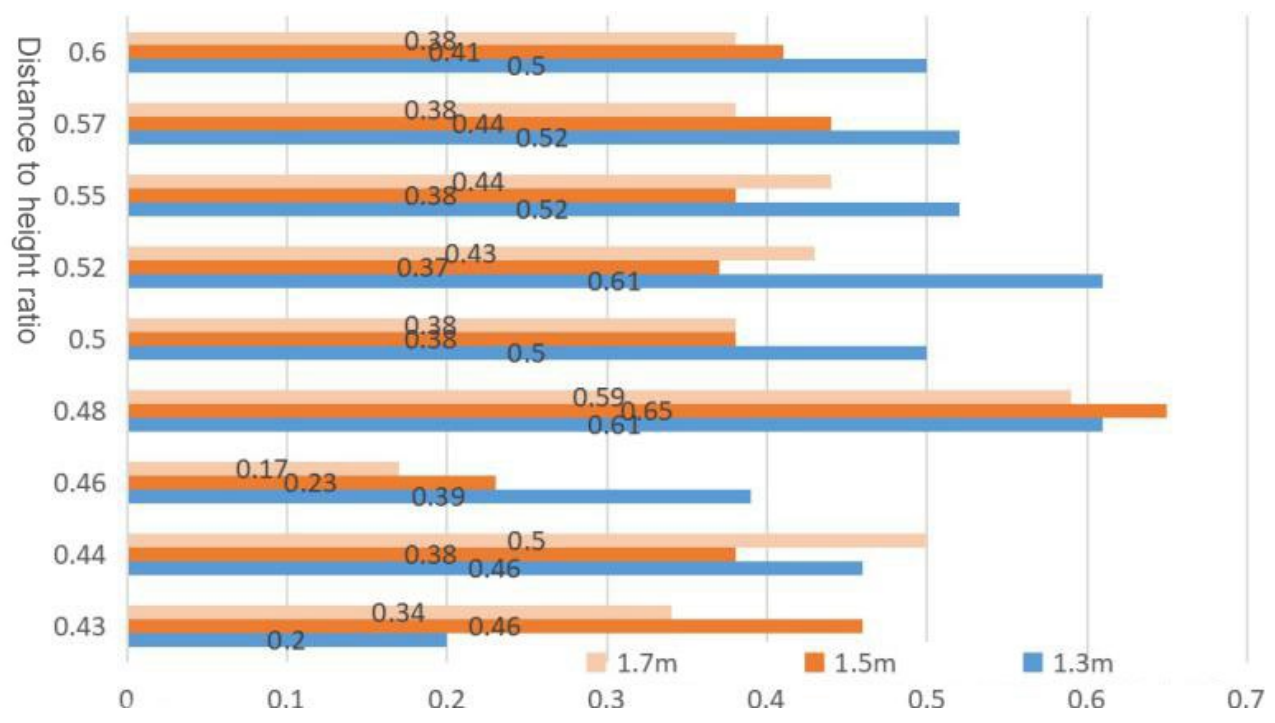

Figure.3. Effect of different distance and height ratios on uniformity

\subsection{Lighting arrangement}


A total of two ways of lighting layout to carry out simulation tests, mainly meet the requirements :1.working face illumination is not less than $500 \mathrm{~lx}$, select the three working face on the minimum difference between the test data 2. the three working face illumination uniformity difference is the minimum 3. the appropriate direction of light 4.light source installation capacity reduced to the minimum 5.layout neat and beautiful ${ }^{[1]}$.

Two kinds of arrangement schemes are used to compare, uniform arrangement and cross arrangement, as shown in the following figure, the number of lamps used is 48 by using the spatial coefficient method. The advantages of this scheme are neat and beautiful layout, high utilization rate of lamps and small capacity. However, the disadvantage is that the light source irradiation on the vertical surface is not uniform, the illumination uniformity and illumination uniformity of the three working faces are quite different, and the plants will appear the phenomenon of unsaturated at the top of the canopy. The biggest advantage of cross arrangement is that it can solve the problem of uniform arrangement, which can significantly improve the situation of large difference in working face ${ }^{[4]}$. The disadvantage of this scheme is that the installation capacity of lamps is large and the installation is not convenient.

The dialux evo simulation lamp is uniform, the arrangement mode is $6 \times 8$, the rotation angle of the lamp is $0^{\circ}$, and the installation position is directly above the strawberry plant $(2.5 \mathrm{~m}$ from the ground). According to the simulation results, the illumination of the three working faces is $1 x$ 、 475lx、537lx.:306 The illumination uniformity is $0.5,0.47,0.24$, respectively. The higher the illumination is, the greater the ratio of distance to height is, so the low illumination uniformity can not guarantee the significantly reduced illumination difference in the three working faces. The use of cross-cloth lamp increases the number of lamps, improves the illumination and illumination uniformity of the working face, so in the same case, the luminous angle of the lamp is 0 degrees, and the difference between the three working faces is obviously improved. So choose cross-cloth lamp. 


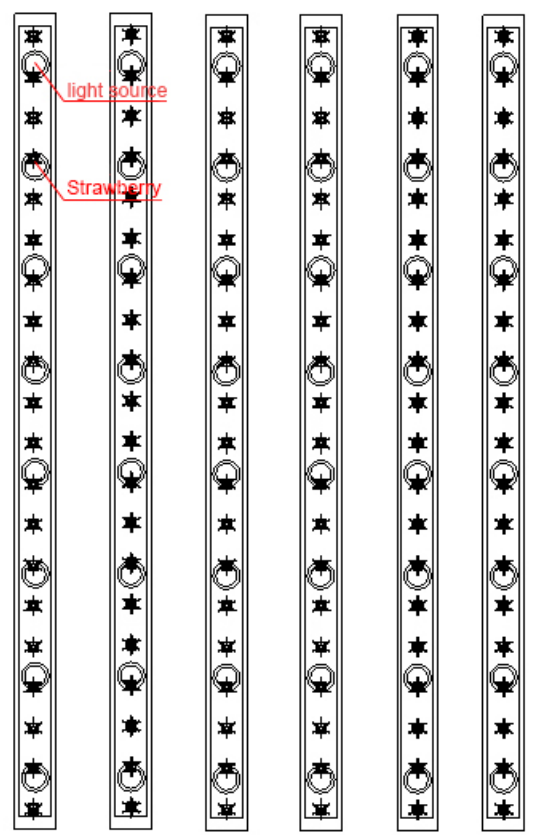

Figure.4. Uniform layout of lamps

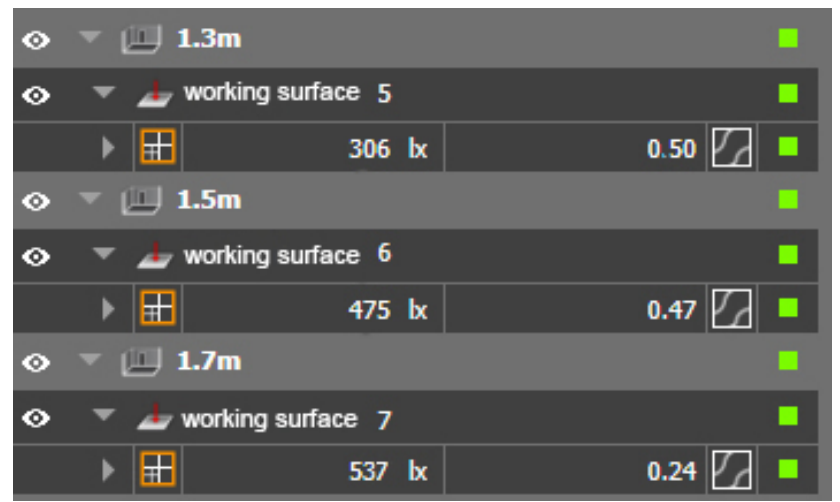

Figure.5.Illumination and illumination uniformity of the working surface with uniform lighting 


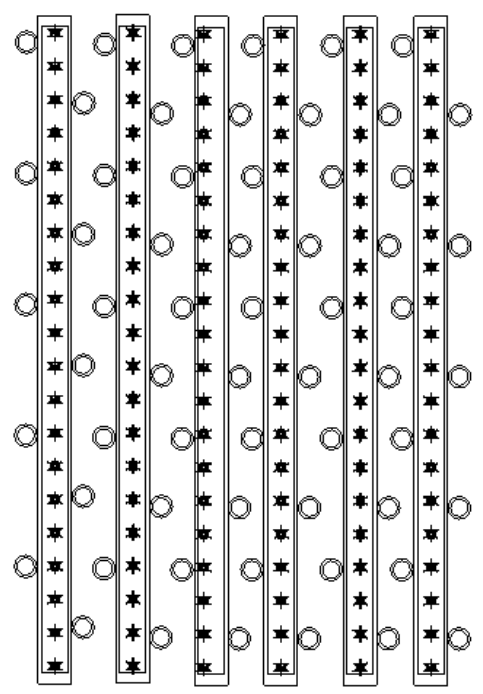

Figure.6. Cross-cutting arrangements

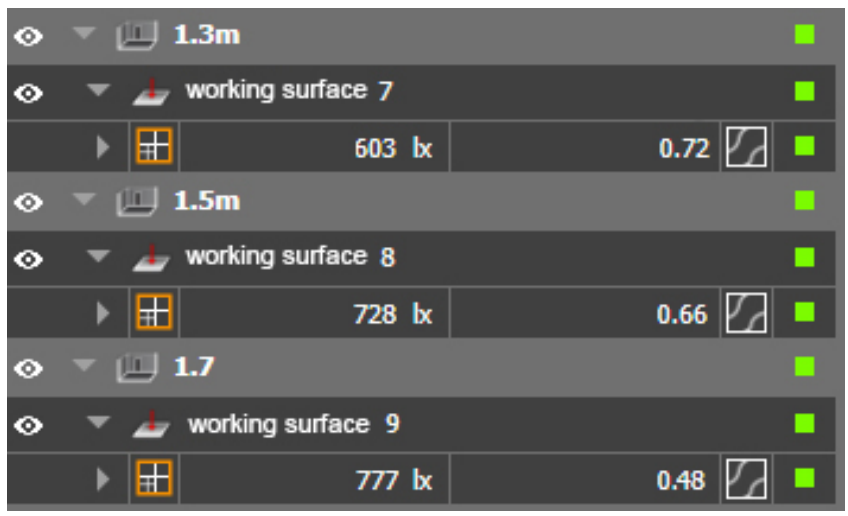

Fig.7. Illumination and illuminance uniformity of working surface with cross-lights 


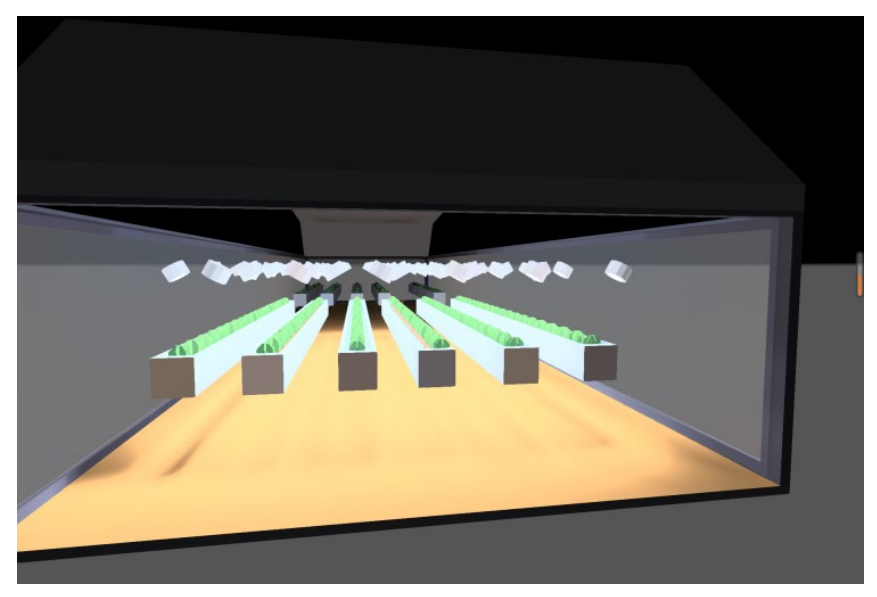

Figure.8. Lamp installation diagram

\subsection{Luminescence angle}

It is found that the shading problem between different layers of fixed cultivation frame is improved to some extent.The direction of light irradiation is parallel to the direction of cultivation frame and the direct light is used to the maximum extent. The main problem of this method is that the upper layer of the same planting frame shading the lower layer, which will lead to different layers of strawberry lighting environment inconsistent, affecting its yield and quality so do not change the way of plant cultivation ${ }^{[2]}$. Whether changing the light source installation angle is also a solution. Therefore, we carry out simulation experiments, because in vertical cultivation.Experimental Environment Reference ${ }^{[5]}$, the tilt angle range of the plant is $20-50^{\circ}$, which can guarantee the reasonable installation capacity of lamps and lanterns, so the same range is selected at the luminous angle of the light source. A total of 16 groups of experimental data $20^{\circ}-50^{\circ}$ were increased in $2^{\circ[3]}$ At the same time, in order to increase the uniformity of the working face, avoid the adjustment of the left and right sides of the plant due to the luminous angle. 


\section{Simulation results and analysis}

Using dialux evo simulation, the whole length of greenhouse space is $28 \mathrm{~m}$, wide $8 \mathrm{~m}$, the whole is divided into left and right parts, and symmetrical, only one side is tested in the test. Strawberries evenly placed in six rows at intervals of $0.6 \mathrm{~m}$. The lamp installation height is 2.5 musing the cross cloth lamp way, carries on the simulation test from $20^{\circ}$ to $50^{\circ}$, the test result arranges the data as follows:

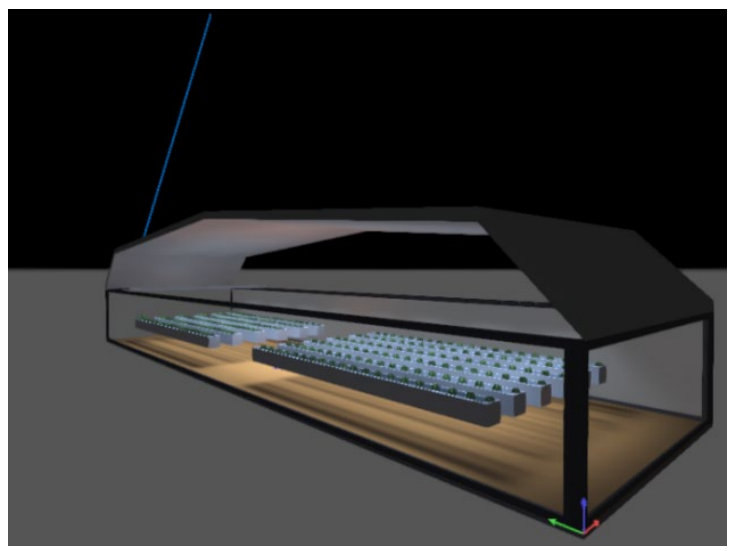

Fig .9. Simulation results of greenhouse

Table .1. Illumination and uniformity of lamps and lanterns in three working faces at different angles

\begin{tabular}{|c|c|c|c|c|c|c|c|c|c|c|c|c|c|c|c|c|c|}
\hline & & 20 & 22 & 24 & 26 & 28 & 30 & 32 & 34 & 36 & 38 & 40 & 42 & 44 & 46 & 48 & 50 \\
\hline \multirow{3}{*}{ Illumination } & $1.3 \mathrm{~m}$ & 599 & 590 & 585 & 579 & 566 & 564 & 555 & 554 & 502 & 491 & 474 & 430 & 430 & 401 & 359 & 368 \\
\hline & & & & & & & & & & & & & & & & & \\
\hline & $1.5 \mathrm{~m}$ & 682 & 682 & 669 & 659 & 643 & 646 & 606 & 588 & 520 & 520 & 477 & 476 & 434 & 429 & 392 & 371 \\
\hline \multirow{2}{*}{$\mathrm{lx}$} & $1.7 \mathrm{~m}$ & 748 & 741 & 727 & 704 & 691 & 686 & 637 & 612 & 598 & 597 & 569 & 566 & 519 & 474 & 427 & 399 \\
\hline & $1.3 \mathrm{~m}$ & 0.69 & 0.67 & 0.60 & 0.55 & 0.55 & 0.65 & 0.65 & 0.65 & 0.64 & 0.57 & 0.59 & 0.61 & 0.61 & 0.50 & 0.52 & 0.52 \\
\hline \multirow[t]{2}{*}{ Uniformity } & $1.5 \mathrm{~m}$ & 0.46 & 0.38 & 0.54 & 0.54 & 0.61 & 0.67 & 0.60 & 0.59 & 0.59 & 0.51 & 0.43 & 0.37 & 0.41 & 0.38 & 0.39 & 0.39 \\
\hline & $1.7 \mathrm{~m}$ & 0.24 & 0.37 & 0.37 & 0.49 & 0.48 & 0.66 & 0.55 & 0.59 & 0.46 & 0.37 & 0.22 & 0.43 & 0.44 & 0.38 & 0.28 & 0.20 \\
\hline
\end{tabular}

When the luminous angle is less than $30^{\circ}$, the difference between the three working faces 
decreases linearly with the increase of luminous angle, but the change is significant but the luminous angle is more than $30^{\circ}$. The gap between the three working faces also increases, can be said that $30^{\circ}$ is a critical value. Therefore, the most suitable luminous angle at the same installation height should be $30^{\circ}$. At an angle of $30^{\circ}$, the illuminance of its three working faces is :564、646、 686. In terms of illuminance and uniformity, the illuminance of the three working faces is the smallest in 16 groups of experiments, and the maximum difference of illuminance uniformity is only 0.02 . To sum up, this scheme is the optimal scheme at present.

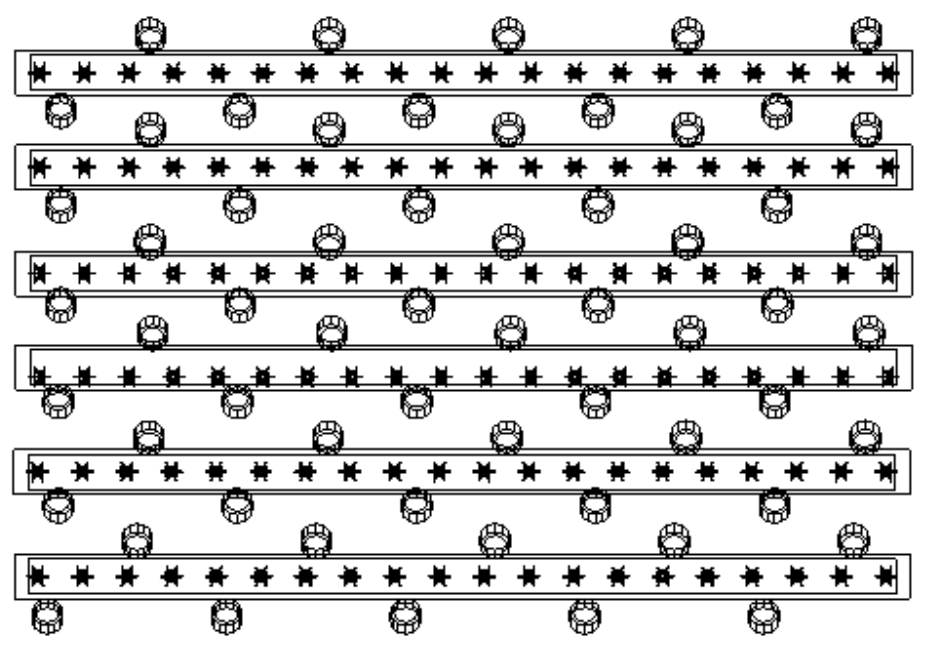

Figure.10. Cross arrangement of lamps and lanterns 

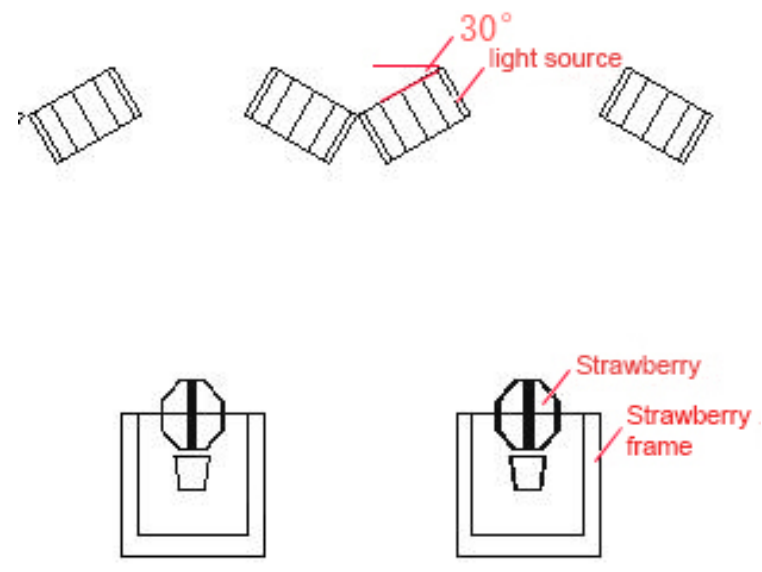

Figure .11. Left view of lighting method

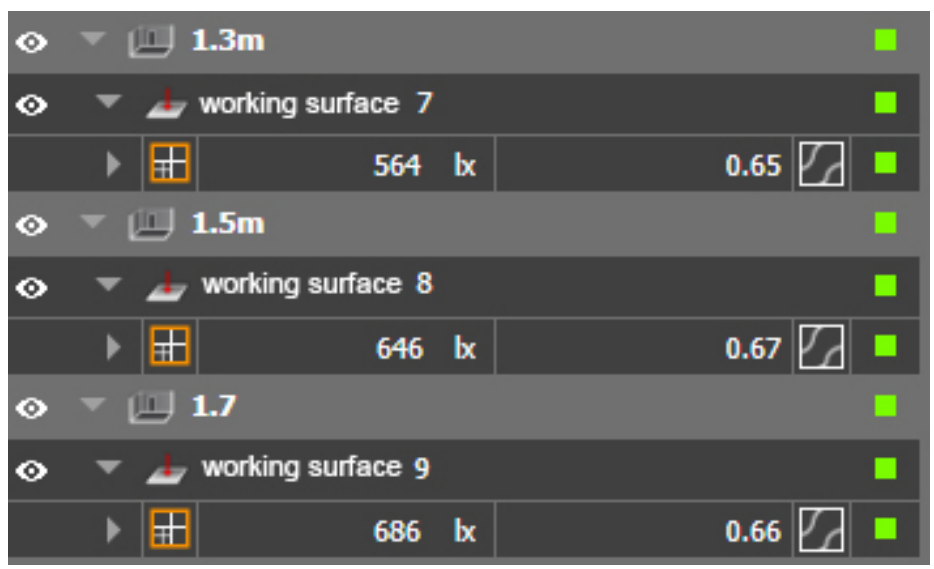

Figure.12. Simulation results at $30^{\circ}$ angle

\section{5 bundles}

When the installation height and arrangement mode are determined, when the luminous angle is less than $30^{\circ}$, the difference between the three working faces decreases linearly with the 
increase of luminous angle, but when the luminous angle is more than $30^{\circ}$, the gap between the three working faces increases with the increase of luminous angle $30^{\circ}$ and the installation height is $2.5 \mathrm{~m}$. The cross-arrangement method used as the light environment design scheme of strawberry shed, and the optimized simulation parameters are obtained. The illumination of three high working faces is $5641 \mathrm{x} 、 6461 \mathrm{x} 、 6861 \mathrm{x}$. respectively The uniformity of illumination was $0.65 、 0.67$ and 0.66 respectively.

A systematic study has been carried out in this experiment on LED light supplement, and the most suitable installation method for strawberry plant light supplement has been obtained, which provides a theoretical basis for the production and application of sunlight greenhouse light supplement in practical strawberry planting. Generally speaking, the LED and light supplement in the solar greenhouse not only ensures the yield of production, but also improves the quality of strawberry fruit and is suitable for production. Now in agricultural production, the solar greenhouse in our country has been greatly popularized and applied, and the LED light-filling technology will become more and more important ${ }^{[6]}$. LED light-filling can accurately regulate the growth and development of plants and their fruit, and can actively regulate plant growth if properly applied. In agricultural production, we can obtain high value and high return agricultural products according to different plant demand for light. At the same time, it also meets the requirements of high efficiency and high yield of scientific and technological agriculture in modern society, and has a very good prospect of application and popularization. 


\section{References}

[1] Lingling Wei, Qichang Yang,Shuili Liu . Research Status and Application Prospect of LED in Plant Factories [J]. China Agronomy Bulletin ,2007,23(011):408-411.

[2] Chunling Wang,Weitang Song,Shumei Zhao,etc. Improving the Light Temperature Environment and Improving Strawberry Yield [J].with Japanese Strawberry Stereo Cultivation Frame Journal of Agricultural Engineering ,2017,33(011):187-194.

[3] Weitang Song .Shumei Zhao . A three-dimensional strawberry cultivation frame for improving light temperature environment and increasing strawberry yield in Quming Mountain [Journal of J] Agricultural Engineering (2017)

[4] Zhengmin Di, Dongxiang Yu , Jianfeng Zhang, etc. [J]. on Vegetable Production and Management Technology for Haze Weather Facilities Chinese melon and vegetable ,2016,29(02):44-45.

[5] Jun Liu,Conghua Xie. The invention relates to an efficient method for producing potato trial tube potato and a culture box thereof [P],china:CN02131125.0,2004-04-14.

[6] Xueyi Zhu,Juan Tang,Muqing Liu, etc. A LED Remote Lighting System for Promoting the Growth of Dendrobium candidum[P],china:CN201520265016.5,2015-10-07. 